\title{
Los fenómenos psicológicos no investigados en la comunicación educativa
}

\author{
Emilio Alberto Ortiz-Torres*
}

Doctor en Ciencias Psicológicas. Profesor titular, Centro de Estudios de Educación, Universidad de Holguín, Cuba. Correo electrónico: eortiz@ict.uho.edu.cu

Recibido: 25 de enero del 2016 Aprobado: 13 de septiembre del 2016

Cómo citar este artículo: Ortiz-Torres, E. A. (2017). Los fenómenos psicológicos no investigados en la comunicación educativa. Pensando Psicología, 13(21), 71-80. doi: http://dx.doi.org/10.16925/pe.v13i21.1715

\section{Resumen}

Propósito: el artículo tiene por objetivo valorar la importancia del estudio científico de los fenómenos psicológicos de la sugestión, la percepción interpersonal y el rumor, los cuales inciden en la comunicación educativa. Tema: el tema que se trabaja es la existencia e influencia de dichos fenómenos subjetivos en los diferentes contextos formativos en los que se desarrolla la comunicación interpersonal. Desarrollo: se realiza un análisis teórico sobre la comunicación educativa como concepto interdisciplinario, por las confluencias existentes entre la psicología y la educación como disciplinas independientes, con su correspondiente contenido y extensión. Sus fundamentos teóricos generales reflejan las diferentes posturas epistemológicas que la sustentan, de lo que se infiere la riqueza de las concepciones particulares existentes que han propiciado la obtención de resultados científicos novedosos y con amplia aplicación en la práctica educativa. Sin embargo, los investigadores no han llegado a profundizar en el estudio de fenómenos existentes en todo proceso comunicativo (como lo son el nivel intrapersonal, la sugestión, la percepción y el rumor, los cuales están presentes dentro de dicho proceso comunicativo en el ámbito escolar), al considerarlos no significativos o improcedentes. Conclusiones: a partir de la valoración realizada, se destaca la importancia de estos fenómenos presentes y latentes en las diferentes manifestaciones de la comunicación educativa, así como la pertinencia de estudiar sus peculiaridades y las potencialidades para incorporarlos de manera intencional en el perfeccionamiento de la comunicación en el campo educativo.

Palabras clave: comunicación educativa, comunicación interpersonal, comunicación intrapersonal, rumor, sugestión, percepción interpersonal. 


\title{
Unresearched psychological phenomena in educational communication
}

\begin{abstract}
Purpose: The article aims to assess the importance of the scientific study of suggestion, interpersonal perception and rumor as psychological phenomena that affect educational communication. Topic: The subject that is being addressed is the existence and influence of such subjective phenomena in different educational contexts in which interpersonal communication takes place. Development: A theoretical analysis is carried out on educational communication as an interdisciplinary concept, due to existing confluences between psychology and education as independent disciplines, with its respective contents and scope. Its general theoretical foundations reflect the different epistemological positions that support it, from which we infer the richness of existing particular conceptions that have led to the attainment of novel scientific results and are widely applicable to educational practice. However, researchers have not delved into the study of phenomena present in any communicative process (such as intrapersonal level, suggestion, perception and rumor, which are present in such communicative process in the educational field), but are considered to be not significant or improper. Conclusions: Based on the assessment made, the importance of these present and latent phenomena in different expressions of educational communication are emphasized, as well as the relevance of studying their peculiarities and potentialities to intentionally incorporate them in the improvement of communication in the educational field.
\end{abstract}

Keywords: educational communication, interpersonal communication, intrapersonal communication, rumor, suggestion, interpersonal perception.

\section{Os fenômenos psicológicos não investigados na comunicação educativa}

\section{Resumo}

Propósito: este artigo tem por objetivo avaliar a importância do estudo científico dos fenômenos psicológicos da sugestão, da percepção interpessoal e do rumor, os quais incidem na comunicação educativa. Tema: o tema que se trabalha é a existência e a influência desses fenômenos subjetivos nos diferentes contextos formativos nos quais se desenvolve a comunicação interpessoal. Desenvolvimento: realiza-se uma análise teórica sobre a comunicação educativa como conceito interdisciplinar, pelas confluências existentes entre a psicologia e a educação como disciplinas independentes, com seu correspondente conteúdo e extensão. Seus fundamentos teóricos gerais refletem os diferentes posicionamentos epistemológicos que a sustentam, do qual se infere a riqueza das concepções particulares existentes que têm propiciado a obtenção de resultados científicos inovadores e com ampla aplicação na prática educativa. Contudo, os pesquisadores não têm aprofundado no estudo de fenômenos existentes em todo o processo comunicativo (como o nível intrapessoal, a sugestão, a percepção e o rumor, os quais estão presentes dentro desse processo comunicativo no âmbito escolar), ao considerá-los não significativos ou improcedentes. Conclusões: a partir da avaliação realizada, destaca-se a importância desses fenômenos presentes e latentes nas diferentes manifestações da comunicação educativa, bem como a pertinência de estudar suas peculiaridades e as potencialidades para incorporá-los de maneira intencional no aperfeiçoamento da comunicação no campo educativo.

Palavras-chave: comunicação educativa, comunicação interpessoal, comunicação intrapessoal, rumor, sugestão, percepção interpessoal. 


\section{Introducción}

La comunicación educativa constituye un término con identidad propia dentro del proceso formativo. La comunidad científica lo acepta como concepto con su correspondiente contenido y extensión, en dependencia de los fundamentos teóricos generales asumidos, ya que las principales corrientes psicológicas contemporáneas (el conductismo, el psicoanálisis, el humanismo, la epistemología genética y la psicología de orientación marxista), lo han abordado de manera peculiar desde sus respectivos enfoques. Esta heterogeneidad epistemológica ha sido una oportunidad valiosa para el abordaje de este complejo fenómeno desde diferentes concepciones, con el aporte de conocimientos científicos novedosos y amplia aplicación en la práctica educativa mundial.

En América Latina, las investigaciones sobre la comunicación educativa han tenido la impronta de dichas corrientes psicológicas contemporáneas, pero no se han limitado a reproducir lo realizado en otras áreas geográficas. Por el contrario, han enriquecido la teoría y la práctica gracias a la originalidad de los aportes realizados por parte de autores tales como Freire (1979, 1980, 1985, 1998); Kaplún (1983, 1984, 1987, 1993); Casañas y Domínguez (1987); Rodríguez (1989); González (1989); González-Rey (1995); Fernández-Durán y Álvarez (1995); Prieto (1998); Ortiz (2001); Karam (2005); Ortiz y González (2012).

Los aportes de estos investigadores, referidos a su relación con la enseñanza y con la actividad, el valor del diálogo, su influencia en el desarrollo de la personalidad, su papel mediador y el papel del grupo, contienen elementos originales que, sin negar las experiencias obtenidas en otras latitudes, destacan la importancia de la comunicación educativa como proceso de intercambio de información, de carácter democrático y participativo, lo cual contribuye de manera significativa al desarrollo de la personalidad de educandos y educadores en la diversidad de los contextos sociales, culturales y políticos de los países latinoamericanos, junto con la incorporación de las tecnologías de la información y la comunicación.

En la actualidad, coexisten múltiples investigaciones culminadas y en proceso a través de proyectos, tesis de maestría y doctorales que abordan directamente la comunicación educativa como problema de permanente continuidad, con miras al perfeccionamiento constante de la educación contemporánea en los diferentes niveles de enseñanza. Todo esto junto con la contribución de modelos, concepciones didácticas, técnicas, estrategias, metodologías, habilidades, competencias, oratoria, comunicación verbal y no verbal, lo cual ha contribuido a elevar su efectividad.

No obstante, se constata una reiteración de temas que, si bien en un momento determinado contribuyeron a su enriquecimiento teórico, metodológico y práctico, en estos momentos refleja un agotamiento creativo en razón a la redundancia de contenidos abordados. Sin embargo, existen otros fenómenos y procesos importantes apenas estudiados, presentes y con frecuencia latentes en toda comunicación interpersonal en general, y en la comunicación educativa en particular. El objetivo del artículo es valorar la importancia del estudio científico de varios fenómenos psicológicos que inciden en la comunicación educativa, así como sobre los que no hay suficientes criterios científicos - ya que no han sido estudiados por los investigadores-, tales como la comunicación intrapersonal, la sugestión, la percepción interpersonal y el rumor.

\section{Desarrollo}

\section{La comunicación intrapersonal}

En toda concepción teórica subsisten, de manera latente o explícita, tres niveles de análisis perfectamente discernibles y delineados en la literatura especializada: el nivel teórico general, el teórico particular y el metodológico de la investigación (Ortiz, 2012). Estos niveles manifiestan el carácter de sistema que toda concepción científica debe tener en su intento de reflejar la realidad en sus elementos más esenciales, desde las posiciones más generales, hasta las prescripciones de cómo estudiar rigurosamente su objeto y su correspondiente impacto.

Sobre el nivel teórico general y particular de la comunicación existe una abundante literatura que, desde el siglo xx, aborda sus referentes filosóficos y epistemológicos, en correspondencia con las corrientes psicológicas contemporáneas que le sirven de base, como, por ejemplo, Engels (1973); Vigotsky (1980); Corrile (1981); Zabalza (1984); Rogers (1985); González-Rey (1985, 1995); Bodalev (1986); Martin-Serrano (1986); Piñuel (1986); Watzlawick, Blanin y Jackson (1987); IbáñezMartin (1987); Colectivo de Autores (1989); Lomov (1989); Asensio (1991); Potrony (1992); y Durán (1996).

Watzlawick et al. (1987) aportaron un principio fundamental: la imposibilidad de no comunicar, y Lomov $(1983,1989)$ una serie de principios sobre la comunicación con carácter teórico general también: 
- La comunicación no se reduce al lenguaje verbal, porque todo el organismo es instrumento de ella.

- No se restringe a la mera transmisión de información, no solo se trasmite, se crea dentro del propio proceso comunicativo.

- En la comunicación se resuelve la contradicción entre lo particular y lo general de los hombres, entre sus cualidades generales y particulares.

- El hombre se realiza y asimila en la comunicación su esencia general.

Asimismo, existen concepciones en cuanto al objeto de estudio dentro de las investigaciones denominadas multinivel y planteadas por Mugny y Doise (1991); González y Peiró (1992); Gaviria (1992); Arróspide y Cerrato (1996).

En las investigaciones que tienen por objeto el proceso educativo escolarizado es importante distinguir y delimitar explícitamente qué nivel o niveles se estudian para la determinación del alcance de los resultados científicos y sus correspondientes generalizaciones. En ellas están presentes los niveles de influencia social, tales como lo son el personal (subjetivo), interpersonal (intersubjetivo), el grupal y el comunitario. Cada uno está bien definido desde el punto de vista científico, y tiene la posibilidad de ser abstraído como objeto de investigación independiente, aunque es totalmente imposible aislar o evitar las interacciones y confluencias que existen entre ellos, dada su sistematicidad y complejidad.

Las investigaciones sobre la comunicación educativa han enfatizado y reiterado los niveles interpersonal, grupal y comunitario, pero el nivel personal (intrapersonal) apenas ha sido abordado, ya que resulta esencial en razóna a su fuerte raigambre subjetiva y su papel determinante en la comunicación del sujeto con las demás personas y grupos humanos con los que interactúa. Si bien en el orden histórico la comunicación interpersonal preexiste a la intrapersonal, una vez que esta surge gracias al desarrollo de la autoconciencia del sujeto, se erige en determinante con respecto a las existentes en los otros niveles. El estudio del nivel intrapersonal de la comunicación educativa revelaría resultados científicos interesantes, novedosos y enriquecería los conocimientos existentes sobre ella.

La comunicación intrapersonal posee un carácter sui géneris, pues la persona se dicotomiza en dos sujetos dentro de sí. En esta comunicación predomina el lenguaje interior y prima el sentido sobre el significado, ya que el contenido adquiere un valor semántico solo para él y con carácter predicativo. Ocurre de manera constante y puede adoptar diferentes formas, las cuales van desde el diálogo hasta el soliloquio, ya que, incluso a solas, los seres humanos conservan las funciones de la comunicación.

Para determinadas personas la comunicación intrapersonal adopta variantes tan intensas que se erigen en elementos autorreguladores de la personalidad (como resultado de su influencia), lo cual está asociado al desarrollo de un alto nivel de criticismo y creatividad.

El monólogo está fuertemente vinculado a esta comunicación peculiar, al ser considerado una construcción psicológica relativamente tardía desde el punto de vista ontogenético, con carácter voluntario y consciente a causa de los procesos psíquicos implicados.

En determinadas ocasiones esta modalidad comunicativa posee peculiaridades que se integran a las cualidades profesionales necesarias para el éxito de la actividad, en la cual la creatividad es algo fundamental, como lo es en el caso de los científicos, los artistas y los profesores. Ellos desarrollan un intenso proceso comunicativo interno como paso previo para el acto creador, y de acuerdo con las cualidades individuales, este adquiere matices originales.

Las dificultades metodológicas en la investigación de la comunicación intrapersonal son evidentes, pero es viable a través de personas creativas en diferentes actividades profesionales dentro de la metodología de estudios de caso, destacadas por varios autores en las investigaciones para corroborar hipótesis y posiciones teóricas (Rodríguez, Gil y García, 1999; Flyvbjerg, 2004; Martínez, 2006; y Stake, 2007).

El carácter inconsciente que regularmente tiene la comunicación intrapersonal para las personas, condiciona la necesidad de desarrollar actividades previas de sensibilización y concientización al fenómeno que será sometido a un análisis introspectivo. Este autoanálisis individual debe combinar reflexiones de vivencias anteriores con situaciones problemáticas planteadas por el investigador, de manera intencional y experimental, como vía para corroborar su dinámica peculiar y subjetiva, lo cual permita también la contrastación ulterior de los resultados de la calidad de las actividades creativas realizadas.

Como la comunicación intrapersonal se complementa con la interpersonal, es procedente también caracterizar la personalidad de dichos sujetos, especialmente en su vida relacional. El enfoque ontogenético de la dinámica y evolución de esta comunicación aportaría, 
como parte del estudio de caso, resultados promisorios en aras de profundizar en sus peculiaridades.

El estudio científico de la comunicación intrapersonal permitiría brindar respuestas a los siguientes interrogantes:

- ¿Cuáles son sus peculiaridades dentro de la comunicación educativa?

- ¿Cómo se complementa con la comunicación interpersonal en el contexto formativo?

- ¿Cómo podría perfeccionarse en función de elevar la efectividad de la comunicación educativa?

\section{La sugestión}

Es uno de los medios sociopsicológicos de influencia oral sobre la subjetividad de las personas, dirigido a convencer sin suficientes argumentos, y el cual logra influir en la subjetividad de los demás sin necesidad de un proceso de argumentación racional. Está muy influida por la autoridad y el prestigio del que sugestiona. Se encuentra en todos los contextos de la vida cotidiana y profesional en los que se desarrolla la comunicación interpersonal oral, dada su influencia en las personas a través del contacto oral cotidiano, en un nivel predominantemente afectivo.

La sugestión es el modo de influencia comunicativa calculado para la percepción no crítica de las informaciones, de las cuales se afirma o se niega algo sin demostración. Tiene especial importancia en este proceso el grupo de factores relacionados con la orientación de los interlocutores hacia la personalidad que sugestiona.

El grado de disposición del auditorio tiene gran significación para la sugestión, ya que exige centrar su atención en las informaciones de determinado contenido; percibirlas y asimilarlas, depende en gran medida de las representaciones que este auditorio tenga sobre la persona que desempeña el papel de comunicador, su estatus, conducta, prestigio, la sinceridad de sus intenciones y la realidad de las informaciones que emanan de él.

La sugestión en estado de vigilia es la más importante de las que se realizan en estado de sueño hipnótico y de sueño natural, dado su origen filogénico y por estar más extendida en los diferentes aspectos de la vida social, así como en las actividades de las personas.

Sherkovin (1982) se refiere a la tradicional contraposición errónea de la sugestión con la persuasión, al destacar la importancia de la fuerza sugestiva de la persuasión, ya que ambos fenómenos "no están separados por una pared infranqueable, sino actúan mancomunadamente, en una misma dirección, complementándose mutuamente y elevando, en última instancia, la efectividad informativa de masas" (p. 136). Precisa que la sugestión está determinada no tanto por el contenido de la información, como por su forma exterior, dada su expresividad que enfatiza la significación y el revestimiento emocional del mensaje, así como por el prestigio de la fuente.

González (1989) defiende la sugestión en cuanto procedimiento perfectamente aplicable como recurso de la comunicación, pues es imposible que cada idea que se pretende fijar pueda ser sólidamente argumentada y fundamentada, ya que para ello se necesitarían años, sobre todo en el caso específico de la educación. La eficacia del recurso sugestivo siempre dependerá del prestigio social, de los rasgos de la personalidad, de la sinceridad, de la firmeza e incluso de la apariencia personal del emisor.

Este mismo autor precisa que la sugestión no debe ser considerada como sinónimo de imposición, ya que las personas aceptan las ideas como suyas, las defienden y actúan en consecuencia. Los recursos de la sugestión deben emplearse combinadamente con la persuasión en toda comunicación humana. Considera que en la enseñanza los profesores hacen afirmaciones que no pueden ser demostradas por razones de tiempo, de estructuras de los programas o por el nivel de los estudiantes, quienes aún no tienen las bases para asimilarlas hasta años posteriores, por lo que la sugestión deviene en un recurso didáctico.

La sugestión se manifiesta en el aula de manera constante y hasta inconsciente entre el profesor y sus alumnos, es prácticamente imposible impedirla, así como que sea la persuasión el único recurso efectivo que se utilice. Además, la propia esencia cognitiva y afectiva de la comunicación interpersonal presupone que los mecanismos racionales coexistan con los afectivos. Los profesores están combinando constantemente los recursos de la persuasión y la sugestión en el logro de su labor formativa, la mayoría de las veces de manera espontánea, ya que no todas sus intervenciones e influencias orales están sustentadas en argumentos racionales; hay afirmaciones que carecen de ellos y tienen un impacto en los alumnos por su autoridad, su prestigio y la función que cumplen como docentes.

Sin embargo, en las investigaciones sobre comunicación educativa la sugestión apenas ha sido tenida en cuenta, debido a prejuicios que la consideran algo negativo que debe ir desapareciendo a medida 
que se incrementa el nivel cultural de los educandos. En la realidad educativa es imposible excluirla, porque forma parte de la propia esencia de la comunicación interpersonal.

Algunas de las sugestiones más comunes realizadas por los profesores en el aula son: "Yo sé que vas a lograrlo", "Vas a salir bien en el examen", "Estoy seguro que tu puedes hacerlo", "No vas a tener problemas, vas a tener éxito", "Este problema va a tener solución". Resultan afirmaciones sin la correspondiente fundamentación persuasiva, pero en determinados momentos tienen un efecto subjetivo inmediato porque brindan seguridad y confianza, por lo que se pueden denominar "sugestiones positivas".

No obstante, también existen sugestiones negativas que provocan el efecto subjetivo contrario y con un posible impacto formativo contraproducente, como, por ejemplo: "Por mucho que te esfuerces al final vas a reprobar", "No confío en tus posibilidades", "No todos se van a graduar".

Asimismo, el estudio de la sugestión dentro de la comunicación educativa constituye un problema apenas estudiado, ya que los investigadores han profundizado en otros fenómenos más racionales y explícitos. Se carece de datos teóricos y empíricos sobre las sugestiones que realizan los profesores en el aula, si las realizan de manera intencional o no, y cuál es su impacto formativo. Esta información podría brindar respuestas científicas a los siguientes interrogantes:

- ¿Cuáles son las peculiaridades de la sugestión en la comunicación educativa?

- ¿Cuál es el impacto formativo de las sugestiones positivas y negativas?

- ¿Cómo se complementa la sugestión con la persuasión en el proceso formativo?

- ¿Cómo podría contribuir la sugestión en la efectividad de la comunicación educativa?

\section{Rumor}

Es uno de los medios socio-psicológicos de influencia oral sobre la subjetividad de las personas, el cual consiste en la trasmisión espontánea de ideas que no precisan su origen, se van tergiversando paulatinamente y están asociadas a determinadas necesidades insatisfechas de las personas, en las que lo informativo y lo afectivo están estrechamente relacionados, provocando diferencias notables entre lo que se orienta y lo que llega a los destinatarios. Se considera, por algunos autores, que constituye el medio de comunicación más antiguo del mundo, dada su trasmisión oral.

Las investigaciones sobre el rumor en la psicología social norteamericana fueron las pioneras desde la primera mitad del siglo $\mathrm{xx}$ - en obtener resultados científicos valiosos que permitían profundizar en este fenómeno comunicativo con un enfoque predominantemente funcionalista, con gran impacto social y de amplia aplicación en la propaganda y en la publicidad a causa de su influencia real en la opinión pública.

Allport y Postman lo definen como proposiciones específicas para creer, las cuales se pasan de persona a persona, por lo general oralmente (aunque pueden ser escritas y radiodifundidas), sin medios probatorios que permitan demostrarlas. La ley básica del rumor tiene dos condiciones esenciales para que prospere: es importante para un sector determinado, y los hechos reales son ambiguos, con pocas noticias o contradictorias (citados por Moya, 1955).

Biain (2013) realiza un análisis histórico sobre el estudio del rumor y precisa que los autores Gordon W. Allport (1897-1967) y Leo Postman (1918-2004) son reconocidos como los primeros en estudiarlo científicamente, y cuyos resultados fueron publicados en Psicología del Rumor. Posteriormente, en 1975 el psicólogo social francés Michel Louis Rouquette (1948-2011) continúa investigándolos en otro contexto político y social, con la publicación de Los Rumores. En la década de los ochenta aparece en Francia Rumores. El medio de difusión más antiguo del mundo, del autor Jean Noël Kapferer.

Sherkovin (1985) se refiere a la importancia del estudio de sus regularidades psicológicas, porque expresan estados de ánimo y opiniones sociales, pero también se ha demostrado que es una de las vías para formar dichos estados de ánimo y opiniones sociales. En los rumores subyacen dos características: expresiva e informativa; la primera refleja el estado emocional de su contenido, y la segunda el grado de veracidad, ya que no todos son totalmente falsos ni totalmente verídicos.

Lizarralde (2013) se refiere a su existencia, en el campo de la comunicación social, debido a la falta de una comunicación a tiempo y con verdades corroborables. Por su parte, Tinoco (2004) aborda el daño que provoca al utilizarlo como instrumento para operaciones psicológicas dirigidas a afectar la reputación de alguien. Asimismo, Gago (2009) destaca el papel de las tecnologías como instrumentos poderosos y efectivos en la trasmisión de los rumores entre las personas. 
Los rumores están presentes también en las instituciones educativas y en las aulas; son los directivos, los profesores y los alumnos los receptores, y a la vez sus emisores, porque son consustanciales a la comunicación interpersonal y, en este contexto, están asociados a las necesidades, las expectativas y las motivaciones del estudiantado y del claustro docente. Sin embargo, son insuficientes las investigaciones que aborden las peculiaridades del rumor en el contexto educativo, sus peculiaridades y su impacto formativo. Sería pertinente profundizar en este tema dentro de la comunicación educativa, a partir de los siguientes interrogantes:

- ¿Cuáles son las peculiaridades del rumor dentro de la comunicación educativa?

- ¿Cómo se relaciona con otros fenómenos comunicativos?

- ¿Cuál podría ser su impacto formativo en el perfeccionamiento de la comunicación educativa?

- ¿Podría ser utilizado intencionalmente en la comunicación educativa?

\section{La percepción interpersonal}

En la estructura de la comunicación interpersonal se encuentra el componente perceptivo, el cual está relacionado con las impresiones subjetivas que se conforman como imagen del otro en el proceso interactivo, la cual no está referida solo a la imagen sensorial de sus características físicas, sino también a sus cualidades subjetivas personales.

Andreieva (1984) aclara que no se debe identificar el término percepción interpersonal con el de percepción social, introducido este último por Bruner en 1947. La percepción interpersonal, si bien se deriva de la social, destaca elementos peculiares desde un enfoque típicamente psicológico que justifica su estudio particular.

La comunicación, como percepción mutua entre las personas, incluye la percepción interpersonal. Cuando el sujeto entra en contacto con otra persona, este es percibido por el otro siempre como personalidad. Las impresiones que surgen durante ese proceso juegan un papel regulador en la comunicación, porque conociendo al otro se va formando el mismo sujeto cognoscente, y la exactitud en la imagen del otro depende de la organización de las acciones acordadas con él.

La representación del otro está muy relacionada con el nivel de autoconciencia. La riqueza de las representaciones sobre uno mismo determina la riqueza de las representaciones que se forma el sujeto sobre el otro; cuanto más se descubre al otro, más completa será la representación de uno mismo.

Las impresiones que surgen durante el proceso comunicativo juegan un papel regulador, porque conociendo al otro se va formando el mismo sujeto cognoscente, y la exactitud en la imagen del otro depende de la organización de las acciones acordadas con él.

Hay una afirmación de Marx que ilustra de manera notable este fenómeno:

Para referirse a sí mismo como hombre, el hombre Pedro tiene que empezar refiriéndose al hombre $\mathrm{Pa}$ blo como a su igual. Y al hacerlo así, el tal Pablo es para él, con pelos y señales, en su corporeidad Paulina, la forma o manifestación que reviste el género hombre. (1973, p. 748).

Bodalev $(1982,1986)$ considera que la percepción interpersonal está constituida por patrones que se actualizan en las personas cuando valoran a otras, se distinguen por su diferente grado de generalización y se manifiestan de diferentes formas, las cuales pueden ser la imagen de una persona concreta, la cual es, a los ojos del individuo, el portador personificado de una u otra cualidad positiva o negativa, o de un grupo de cualidades. Esta clasificación puede desarrollarse de manera consciente o espontánea y se hace más compleja con el desarrollo ontogenético.

El análisis de la toma de conciencia de sí a través del otro incluye la identificación y la empatía. La identificación significa la asimilación de sí al otro. Existe un estrecho vínculo entre identificación y empatía. La empatía se define como medio de comprensión del otro, pero emocional, afectiva. El proceso de comprensión del otro se complica con el fenómeno de la reflexión, la toma de conciencia de cómo él es percibido por el otro en la comunicación.

La psicología social ha sido históricamente protagonista en las investigaciones sobre la percepción interpersonal en el ámbito de la política, la publicidad y la propaganda, con resultados profusos y muy útiles en diferentes esferas de la sociedad (Ash, 1946; Laing, Phillipson y Lee, 1973; Barthey, 1982; Janes y Thibaut, 1990; Penman, 1990).

Durán (1989) y Fernández et al. (1995) destacan en sus investigaciones la importancia de la primera impresión que provocan los profesores ante sus alumnos, el llamado "efecto de halo", así como la imagen impersonal que pueden tener de los mismos, lo cual afecta la comunicación entre ellos. 
Murillo y Becerra (2009) estudian cómo perciben los profesores el clima escolar y sus elementos obstaculizadores. Dentro de las dificultades mayores encontraron los problemas de comunicación que afectan su rol profesional.

Sin embargo, los estudios sobre las percepciones que se forman entre profesores y alumnos en la comunicación educativa están poco estudiadas. Estas imágenes que se van conformando paulatinamente en la interacción cotidiana influyen y determinan la efectividad de la comunicación. Se forman de manera espontánea y no siempre reflejan la riqueza de la personalidad de los participantes en el proceso comunicativo, por diferentes causas y condiciones relacionadas con el contexto educativo en el que ocurren.

- ¿Cuáles son las imágenes que tienen los profesores de sus alumnos y estos de aquellos en la comunicación educativa dentro del aula?

- ¿Cuáles son las peculiaridades de imágenes que cada uno tiene de sí dentro de la comunicación interpersonal?

- ¿Predominan imágenes del otro por sus características físicas, psicológicas o funcionales?

- ¿Cómo perfeccionar la percepción interpersonal entre profesores y alumnos en función de la efectividad de la comunicación educativa?

\section{Conclusiones}

Aunque la comunicación educativa posee un copioso arsenal de resultados científicos obtenidos por la gran cantidad de investigadores que se han dedicado a estudiarla, existen varios fenómenos psicológicos implicados en la comunicación interpersonal que no han sido investigados suficientemente en este campo, lo cuales están omnipresentes de manera latente o no y que influyen en su efectividad. Entre estos se encuentran la comunicación intrapersonal, la sugestión, el rumor y la percepción interpersonal.

Les corresponde a los investigadores que se ocupan de este tema asumirlos como objeto de estudio y, desde sus respectivas posiciones teóricas y metodológicas, descubrir sus peculiaridades y las potencialidades, a fin de incorporarlos de manera intencional en el perfeccionamiento de la comunicación en la educación.

\section{Referencias}

Andreieva, G. M. (1984) Psicología social. Moscú: Universidad Estatal de Moscú.

Arróspide, J. y Cerrato, J. (1996). La perspectiva sociocultural en el estudio del grupo. En S. Ayestarán (Ed.), $E l$ grupo como construcción social (pp. 174-188). Barcelona: Plural.

Asensio, J. (1991). La comunicación como medio educativo. Revista Educar, 18, 41-52. Recuperado de: http://educar.uab.cat/article/download/497/480

Asch, S. (1946). La Percepción. Madrid: U.N.E.D.

Barthey, S. H. (1982). Principios de percepción. Ciudad de México: Trillas.

Biain, M. (2013).La comunicación y sus laberintos. Consideraciones acerca del rumor. Recuperado de http://e-marc. net/2013/wp-content/uploads/2013/05/ La-comunicaci\%C3\%B3n-y-sus-laberintos-Emarc-2013.pdf

Bodalev, A. (1982). La percepción del hombre por el hombre. Moscú: Editorial de la Universidad de Moscú.

Bodalev, A. (1986). El desarrollo de la percepción del hombre por el hombre en la comunicación. En Colectivo de Autores, Antología de la Psicología Pedagógica y de las Edades (pp. 132-137). La Habana: Editorial Pueblo y Educación.

Casañas, A. y Domínguez, L. (1987). La comunicación grupal. En Colectivo de Autores, Investigaciones de la personalidad en Cuba (pp. 172-196). La Habana: Editorial Ciencias Sociales.

Colectivo de Autores. (1989). Temas sobre Actividad y Comunicación. La Habana: Editorial de Ciencias Sociales.

Corrile, W. (1981). Psicología de las relaciones humanas. Barcelona: Editorial Herder.

Durán, A. (1989). El proceso docente-educativo como proceso comunicativo. La Habana: Editorial Pablo de la Torriente.

Durán, N. (1996). Enseñanza y comunicación. España: Eunusa.

Engels, F. (1973). Papel del trabajo en el proceso de transformación del mono en hombre. En F. Engels, Obras Escogidas (pp. 66-79), tomo III. Moscú: Editorial Progreso.

Fernández, A., Durán, B. y Álvarez, M. (1995). Comunicación educativa. La Habana: Editorial Pueblo y Educación. 
Flyvbjerg, B. (2004). Cinco malentendidos acerca de la investigación mediante los estudios de caso. Revista Española de Investigaciones Sociológicas, 106(4), 33-62.

Freire, P. (1979). La educación como práctica de libertad. México: Editorial Siglo XxI.

Freire, P. (1980). Pedagogía del oprimido. México: Editorial Siglo XXI.

Freire, P. (1985). Dialogicidad y diálogo. En Colectivo de Autores, Diálogo en interacción en el proceso pedagógico (pp. 39-47). México: Ediciones El Caballito.

Freire, P. (1998). Pedagogía de la Autonomía (2ª ed.). México: Editorial Siglo XxI.

Gago, P. (2009). La subjetividad y el rumor: del "gran pánico" de 1789 a la "era de la Información". Perspectivas de la Comunicación, 2(1), 53-65. Recuperado de http:// dialnet.unirioja.es/descarga/articulo/3016885.pdf

Gaviria, J. (1992). El enfoque multinivel en la evaluación de sistemas educativos. Revista de Investigación Educati$v a, 17(2), 429-443$.

González, V. y Peiró, J. M. (1992). Técnicas de investigación multinivel en las organizaciones. En M. Clemente (Ed.), Psicología Social. Métodos y Técnicas de Investigación (pp. 349-365). Madrid: Eudema.

González-Rey, F. (1985). La categoría comunicación. Su lugar en el sistema de categorías de la psicología marxista, Revista Cubana de Psicología, 2-3, 13-24.

González-Rey, F. (1995). Comunicación, personalidad y desarrollo. La Habana: Editorial Pueblo y Educación.

González, V. (1989). Profesión: Comunicador. La Habana: Editorial Pablo de la Torriente.

Ibáñez-Martin, J. A. (1987). Planteamiento filosófico-educativo del problema de la comunicación. Revista Española de Pedagogía, 45(177), 295-304.

Janes, E. E. y Thibaut, J. W. (1990). Interpersonal Perception. New York: Freeman.

Kaplún, M. (1983). Hacia nuevas estrategias de comunicación en la educación de adultos. Bogotá: Ediciones Unesco.

Kaplún, M. (1984). Comunicación entre grupos. El método del Cassette-Foro. Centro Internacional de investigación para el desarrollo del ciID. Oficina Regional para América Latina y el Caribe.

Kaplún, M (1987). El aporte de la comunicación a una educación democratizadora. Caracas: Celadec.

Kaplún, M. (1993). Del educando oyente al educando hablante. Perspectivas de la comunicación educativa en tiempos de eclipse. Revista Diálogos de la Comunicación, 37, septiembre. Felafacs. Recuperado de: http://
www.educarchile.cl/P0001/File/01-Del\%20Educando\%20Oyente.pdf

Karam, T. (2005). Una introducción al estudio de la epistemología de la comunicación desde la obra de Manuel Martín Serrano. Cinta de Moebio, diciembre, 24. Universidad de Chile. Recuperado de: www.facso.uchile. cl/ publicaciones/moebio/24/karam.htm

Laing, R. D., Phillipson, H. y Lee, A. R. (1973). Percepción interpersonal. Buenos Aires: Editorial Amorrortu.

Lizarralde, A. (2013). A la falta de comunicación impera el rumor. Más poder local, 15, 34-35. Recuperado de http://dialnet.unirioja.es/descarga/ articulo/4247987. pdf

Lomov, B. (1983). Comunicación y regulación social de la conducta del individuo. En Colectivo de Autores, Aspectos socio-psicológicos de la comunicación (pp. 3-32). Facultad de Psicología. Universidad de La Habana.

Lomov, B. (1989). El problema de la comunicación en psicología. La Habana: Editorial de Ciencias Sociales.

Martin-Serrano, M. (1986). Presentación de la teoría social de la comunicación. Revista Española de Investigaciones Sociológicas, 33, 15-34.

Martínez, C. (2006). El método de estudio de caso. Estrategia metodológica dela investigación científica. Revista Científica Pensamiento y Gestión, 20, 165-193. Recuperado de http://www.redalyc.org/articulo.oa?id=64602005\#

Marx, K. (1973). El Capital. Tomo I. La Habana: Editorial de Ciencias Sociales.

Moya, G. (1955). Estudio psicológico del rumor. Resumen y comentarios al libro de G. W. Allport y L. Postman. Revista de Psicología General y Aplicada, 10(3), 129-136.

Mugny, G. y Doise, W. (1991). Niveles de análisis en el estudio experimental de los procesos de influencia social. Anthropos. Boletín de información y documentación, 27, 77-101.

Murillo, P. y Becerra, S. (2009). Las percepciones del clima escolar por directivos, docentes y alumnado mediante el empleo de «redes semánticas naturales». Su importancia en la gestión de los centros educativos. Revista Educación, 350, 375-399.

Ortiz, E. (2001). La comunicación pedagógica. Argentina: Editorial Libros en Red.

Ortiz, E. y González, V. (2012). La comunicación educativa y los medios de enseñanza en la universalización. Ecuador: Editorial Mar Abierto.

Ortiz, E. (2012). Los niveles teóricos metodológicos de las investigaciones pedagógicas. Revista Cinta de Moebio, 
43, 14-23. Recuperado de: www.moebio.uchile.cl/43/ ortiz.html

Penman, R. (1990). Communication processes and relationships. London: Academic Press.

Piñuel, R. J. L. (1986). Fuentes epistemológicas de la teoría de la comunicación. Revista Española de Investigaciones Sociológicas, 33, 15-34.

Potrony, J. (1992). La actividad y la comunicación: convergencias y divergencias. Revista Cubana de Psicología, $9(2), 81-88$

Prieto, D. (1998). Comunicación educativa en el contexto latinoamericano. Intervención Psicosocial, 3(3), pp. 329-345.

Rodríguez, E. (1989). Comunicación Pedagógica. Revista Tecnología y Comunicación Educativas, 4, pp. 9-15, octubre, México, DF.

Rodríguez, G., Gil, J. y García, E. (1999). Metodología de la investigación cualitativa. Granada: Aljibe.

Rogers, C. (1985). La relación interpersonal en la facilitación del aprendizaje. En Colectivo de Autores, Diálogo e interacción en el proceso pedagógico (pp. 61-70). México:
Ediciones El Caballito.

Sherkovin, Y. A. (1982). Problemas psicológicos de los procesos masivos de información. La Habana: Editora Política.

Sherkovin, Y. A. (1985). Fundamentos de la psicología socialy de la propaganda. Moscú: Editorial Progreso.

Stake, R. (2007). Investigación con estudio de casos ( $4^{\mathrm{a}} \mathrm{ed}$.). Madrid: Ediciones Morata SL.

Tinoco, C. (2004). Dinámica del rumor y operaciones psicológicas de daño reputacional. Anales de la Universidad Metropolitana, 4(2), 155-169. Recuperado de http:// dialnet.unirioja.es/descarga/articulo/4002584.pdf

Vigotsky, L. S. (1980). Pensamiento y Lenguaje. La Habana: Editorial Pueblo y Educación.

Watzlawick, P., Blanin, J. B. y Jackson, D. D. (1987). Teoría de la comunicación humana. Barcelona: Herder.

Zabalza, M. (1984). El análisis de la enseñanza desde el modelo comunicacional. Revista Enseñanza, 2, 9-38. 